\title{
Shell Filling and Exchange Coupling in Metallic Single-Walled Carbon Nanotubes
}

\section{Citation}

Liang, Wenjie, Marc Bockrath, and Hongkun Park. 2002. “Shell Filling and Exchange Coupling in Metallic Single-Walled Carbon Nanotubes." Physical Review Letters88 (12): 126801. https:// doi.org/10.1103/PhysRevLett.88.126801.

\section{Permanent link}

http://nrs.harvard.edu/urn-3:HUL.InstRepos:41534527

\section{Terms of Use}

This article was downloaded from Harvard University's DASH repository, and is made available under the terms and conditions applicable to Other Posted Material, as set forth at http:// nrs.harvard.edu/urn-3:HUL.InstRepos:dash.current.terms-of-use\#LAA

\section{Share Your Story}

The Harvard community has made this article openly available.

Please share how this access benefits you. Submit a story.

Accessibility 


\title{
Shell Filling and Exchange Coupling in Metallic Single-Walled Carbon Nanotubes
}

\author{
Wenjie Liang, Marc Bockrath, and Hongkun Park* \\ Department of Chemistry and Chemical Biology, Harvard University, Cambridge, Massachusetts 02138
}

(Received 7 November 2001; published 6 March 2002)

\begin{abstract}
We report the characterization of electronic shell filling in metallic single-walled carbon nanotubes by low-temperature transport measurements. Nanotube quantum dots with average conductance $\sim(1-2) e^{2} / h$ exhibit a distinct four-electron periodicity for electron addition as well as signatures of Kondo and inelastic cotunneling. The Hartree-Fock parameters that govern the electronic structure of metallic nanotubes are determined from the analysis of transport data using a shell-filling model that incorporates the nanotube band structure and Coulomb and exchange interactions.
\end{abstract}

DOI: $10.1103 /$ PhysRevLett.88.126801

Over the past five years, single-electron transistors of individual molecules [1], nanocrystals [2-4], and singlewalled carbon nanotubes (SWNTs) [5-13] have emerged as a powerful experimental probe of the electronic motion in chemical nanostructures. Electron transport in these structures is strongly influenced by single-electron charging and energy level quantization, and transport measurements provide detailed information on their quantum-level structure [14]. Among chemical nanostructures, metallic SWNTs have served as a model system for transport studies, providing evidence for energy-level quantization [5,6], spin filling [7,8], Luttinger liquid behavior [10], and Kondo physics [11]. Detailed knowledge on the electronic structure of SWNTs is still lacking, however, and such basic information as the ground state electronic configuration remains to be established $[7,8]$.

Here we report a systematic transport study of metallic nanotube quantum dots with conductance $\sim(1-2) e^{2} / h$ [the value of $e^{2} / h$ is $38.8 \mu \mathrm{S}$ or $(25.8 \mathrm{k} \Omega)^{-1}$ ] that enables the characterization of electronic shell filling and exchange coupling in SWNTs. Transport measurements of these nanotube devices reveal a distinct four-electron periodicity for electron addition as well as signatures of the Kondo resonance $[11,15-20]$ and inelastic cotunneling $[11,21]$. These observations are analyzed using an electronic shell-filling model that explicitly incorporates the band structure of SWNTs and exchange and Coulomb interactions between electrons within the Hartree-Fock approximation [22-25]. Specifically, five parameters that completely determine the electronic structure of nanotube quantum dots are obtained from an analysis of the experimental data alone: these parameters include the mean level spacing $\Delta$, the subband mismatch $\delta$, the exchange parameter $J$, the charging energy $U$, and the excess Coulomb energy $\delta U$ to put two electrons into a single level. The energetic contribution of exchange coupling is determined to be $\sim 10 \%-20 \%$ of the single-particle level spacing, in accordance with theoretical predictions [22].

Isolated SWNTs were synthesized by chemical vapor deposition using a procedure reported previously $[12,13,26]$. The chemical vapor deposition method was optimized to synthesize well-isolated SWNTs free from
PACS numbers: 73.22.-f, 73.23.-b, 73.63.Fg

graphitic contamination, which, in turn, enabled the fabrication of nanotube devices with varying contact resistances. Nanotubes with a diameter of $\leq 1 \mathrm{~nm}$ were located relative to alignment marks, and electrical leads were defined by electron-beam lithography by depositing a thin layer of chromium, followed by gold [Fig. 1(a) inset]. Electrical characterization was performed as a function of bias voltage $(V)$ and gate voltage $\left(V_{g}\right)$. The degenerately doped silicon substrate acted as a gate electrode to

(a)

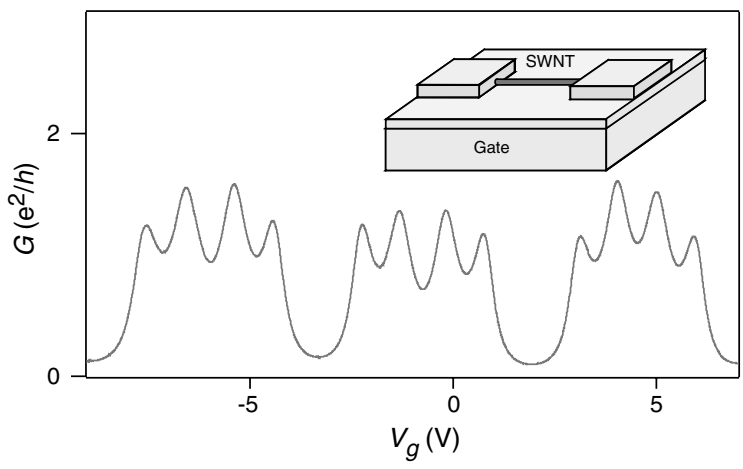

(b)

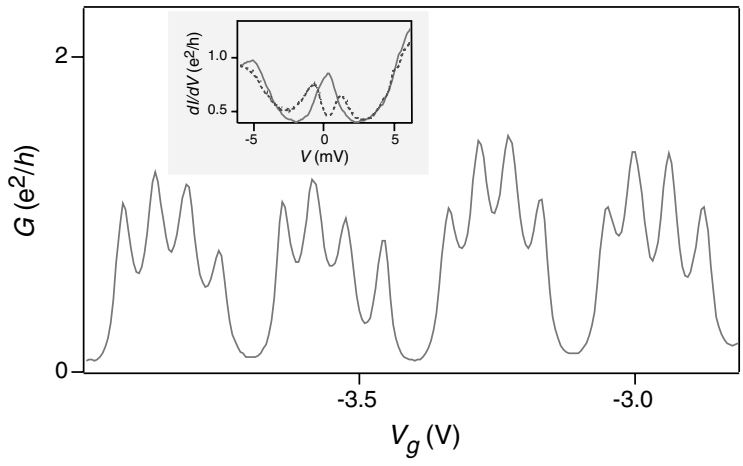

FIG. 1. (a) Plot of linear-response conductance $(G)$ versus $V_{g}$ for a nanotube device with a nanotube length of $\sim 100 \mathrm{~nm}$ at $T=1.5 \mathrm{~K}$. (Inset) Schematic diagram of device geometry. (b) Plot of $G$ versus $V_{g}$ for a nanotube device with a length of $200 \mathrm{~nm}$ at $T=1.5 \mathrm{~K}$. (Inset) A plot of $\partial I / \partial V$ versus $V$ with $V_{g}$ tuned to the first valley of the second four-electron group. The solid and dashed curves correspond to $B=0$ and $9 \mathrm{~T}$, respectively. 
modulate the charge density within the nanotubes. More than 300 nanotube devices were fabricated to date, and they were classified as metallic or semiconducting on the basis of their resistance versus $V_{g}$ behavior at room temperature $[12,13]$. All the observations discussed below pertain to metallic nanotube devices.

Figure 1 shows plots of linear-response conductance as a function of $V_{g}$ obtained from two representative nanotube devices with a $V_{g}$-averaged conductance $\left(G_{\text {av }}\right)$ of $\sim e^{2} / h$. The well-defined conductance peaks in Fig. 1 signify the addition of one electron to the nanotube quantum dot as the dot's electrostatic potential is tuned by varying $V_{g}[6,7,14]$. Remarkably, the conductance peaks form a repeating pattern where the peaks appear in clusters of four, indicating that electron addition to the nanotube exhibits a four-electron periodicity. This behavior has never been observed in previous nanotube quantum-dot studies, and it is in stark contrast from that observed previously in nanotube bundles with $G_{\mathrm{av}} \sim 1.6 e^{2} / h$ [11]. Nearly all $(>10)$ nanotube devices with $G_{\mathrm{av}} \sim(1-2) e^{2} / h$ investigated to date exhibited similar four-electron periodicity, demonstrating its robustness with respect to device to device variation.

Quantitative information on this electron addition pattern can be found from two-dimensional differential conductance $(\partial I / \partial V)-V-V_{g}$ plots in Fig. 2, which show three small conductance gap regions, labeled I, II, and III, followed by a large conductance-gap region labeled IV. This conductance-gap pattern repeats over a wide range $(>20)$ of added electron numbers. The energy $\Delta \mu(N)$ associated with the addition of the $N$ th electron to a nanotube can be obtained by measuring the bias voltage at the upper and lower vertices of the conductance-gap region [14]. Denoting the corresponding $V$ values for each region by $V_{\text {I-IV }}$, the addition energies for the first three electrons are $e V_{\text {I-III }} \sim 4 \mathrm{meV}$, whereas that for the fourth electron is $e V_{\mathrm{IV}} \sim 8 \mathrm{meV}$.

The dark horizontal lines in Fig. 2 that correspond to $\partial I / \partial V$ peaks with $V_{g}$-independent bias voltage positions are additional transport characteristics that are universally observed in nanotube devices with $G_{\mathrm{av}} \sim(1-2) e^{2} / h$. In regions I and III, these dark lines appear at $V=0$, whereas they appear at nonzero bias voltages in region IV. In region II, the $V$ positions of the dark lines vary, depending on the specific four-electron cluster under consideration.

The $\partial I / \partial V$ features appearing at $V=0$ are reminiscent of the Kondo resonance observed previously in quantum-dot [15-20] and nanotube-bundle studies [11]. One important signature of a Kondo resonance is that its bias position splits under a magnetic field $B$. The inset of Fig. 1(b) shows that the $\partial I / \partial V$ peak at $V=0$ indeed splits in $V$ under a magnetic field by $2 g \mu B / e$, where $g=2$ is the electron $g$ factor, and $\mu$ is the Bohr magneton, thus confirming that these features arise from a Kondo resonance. The Kondo resonance is a many-electron phenomenon resulting from the exchange interaction

(a)

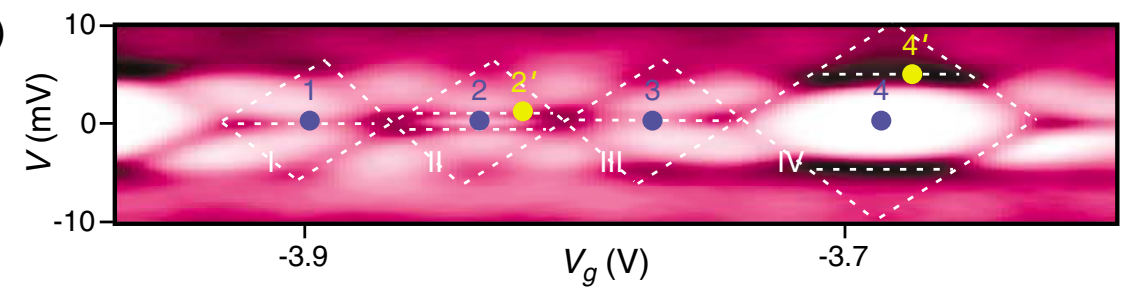

(b)

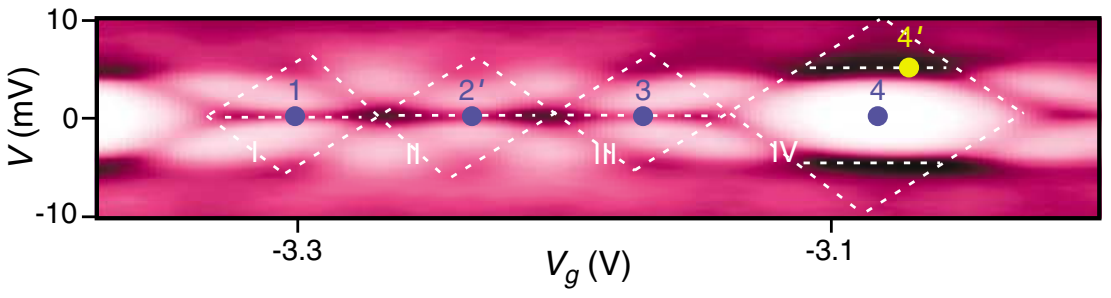

(c)
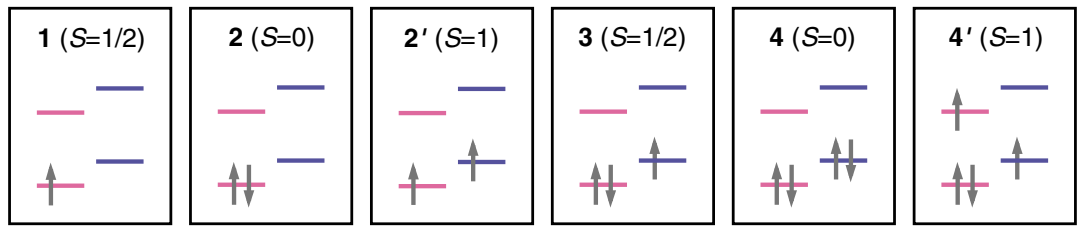

FIG. 2 (color). (a) Two-dimensional color plot of $\partial I / \partial V$ vs $V$ and $V_{g}$ for the nanotube device in Fig. 1(b) at $T=1.5 \mathrm{~K}$. Here the white color corresponds to 0 , and the darkest color corresponds to $1.5 e^{2} / h$. The sloped white dashed lines delineate conductance-gap regions that are marked by I, II, III, and IV, and they are obtained from the theoretical analysis. (b) The same as in (a), but for a different region of $V_{g}$. (c) The diagrams showing the electronic configurations at the positions marked by numbered dots in (a) and (b). Blue and yellow dots indicate the transport features arising from the ground and excited electronic states of the nanotube quantum dot. 
between a localized spin and the conduction electrons in the metallic leads $[15,16]$, and it appears when the electronic state of a quantum dot has nonzero spin $(S)$. The appearance of Kondo resonances within regions I and III therefore indicates that the corresponding electronic ground states have $S>0$. The intermittent appearance of a Kondo resonance in region II suggests that the ground state $S$ value in that region varies with the number of electrons on the nanotube.

The $V_{g}$-independent $\partial I / \partial V$ features at nonzero bias in Fig. 2 suggest, on the other hand, the occurrence of high-order tunneling processes where one electron enters the dot, while another electron simultaneously exits the dot, leaving the dot in an excited state [11,21]. In this inelastic cotunneling process, the bias voltage $V_{\text {in }}$ supplies the excitation energy. Because this excitation does not involve a change in the charge state of the dot, the associated feature in $\partial I / \partial V$ is independent of $V_{g}$, and the value $e V_{\text {in }}$ provides a direct measure of the excited state energy. Upon the application of a magnetic field, the horizontal features in region II shift in their $V$ position and merge into one horizontal line at $V=0$, suggesting a close connection to the Kondo resonance $[11,19,27]$. The horizontal features in region IV, which are far removed from $V=0$, exhibit a broadening of their widths under a magnetic field.

All these observations, including four-electron periodicity, the Kondo resonance, and inelastic cotunneling, can be explained in a unified fashion using a shell-filling model that incorporates the band structure of metallic SWNTs as well as the exchange and Coulomb interactions between electrons [22-25]. Previous theoretical and experimental studies have shown that the electronic properties of metallic SWNTs are determined by two spin-degenerate subbands with linear dispersion [13,28]. In the simplest picture of a nanotube quantum dot, the quantization of these two subbands leads to two sets of spin-degenerate electronic levels with a mean spacing $\Delta$ within each set. The energy mismatch between these two sets of electronic levels can be represented by $\delta(\delta \leq \Delta / 2)$, as shown in the Fig. 3 inset.

This picture can be further refined by including the Coulomb and exchange interactions, represented by three mean field parameters within the Hartree-Fock approximation: the charging energy $U$ that accounts for the long-range Coulomb repulsion between electrons, the exchange energy $J$ that favors spin alignment, and the excess Coulomb energy $\delta U$ to put two electrons into a single level [22]. With these parameters, the Hamiltonian of the nanotube quantum dot can be written as [22]

$$
\begin{aligned}
H= & \sum_{\mu, \sigma, l} \varepsilon_{l \mu} n_{l \mu \sigma}+\frac{1}{2} U\left(\frac{Q_{\mathrm{dot}}-Q_{\mathrm{ext}}}{e}\right)^{2} \\
& +\delta U \sum_{\mu, l} n_{l \mu \uparrow} n_{l \mu \downarrow}+J \sum_{\mu, \mu^{\prime}} N_{\mu \uparrow} N_{\mu^{\prime} \downarrow} .
\end{aligned}
$$

Here $\varepsilon_{l \mu}=l \Delta+(\mu-1) \delta$ is the energy of the $l$ th quantum level originating from the $\mu$ th subband $(\mu=1,2)$,

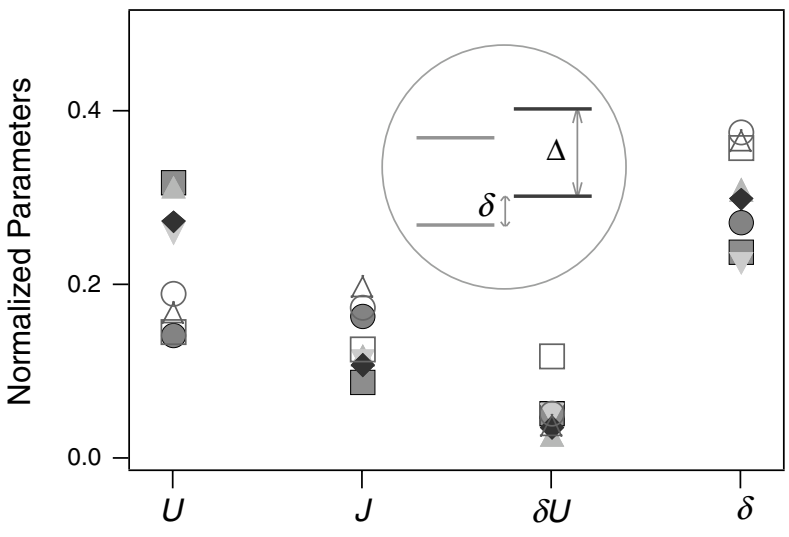

FIG. 3. Parameter values of $U, J, \delta U$, and $\delta$ normalized to $\Delta$ that are extracted from the transport data. Open symbols were obtained from a nanotube device with 100-nm length [Fig. 1(a)], and filled symbols were obtained from a nanotube device with 200-nm length [Figs. 1(b) and 2]. Each shape indicates values obtained from a different four-electron cluster. (Inset) Singleparticle level structure used in our model.

$Q_{\text {dot }}$ is the excess charge on the nanotube, $Q_{\text {ext }}$ is the gate-induced charge on the dot, $n_{l \mu \sigma}$ is the number operator for the $l$ th level with spin $\sigma$ in the $\mu$ th subband, and $N_{\mu \sigma}$ is the total number of electrons with spin $\sigma$ in the $\mu$ th subband. Within this model, the five energy parameters, $\Delta, \delta, U, J$, and $\delta U$ completely determine the energies of all the possible spin-electronic configurations of a nanotube quantum dot [22].

This shell-filling model with two sets of spindegenerate electronic levels provides a natural framework for the four-electron periodicity observed in Figs. 1 and 2. Moreover, the shell-filling model explains the ground state spin configurations observed in Fig. 2 as well. Assuming that $J$ and $\delta U$ are small compared to $\Delta$, two independent spin-filling schemes are possible in the model. In the first scenario, appropriate for $J+\delta U<\delta$, two electrons of opposite spin fill each spin-degenerate level before filling higher energy levels, producing the sequence of ground state spins $S=1 / 2, S=0, S=1 / 2$, and $S=0$. In the second scenario, which occurs when $J+\delta U>\delta$, the ground state for two electrons is a spin triplet, and the sequence of ground state spins becomes $S=1 / 2$, $S=1, S=1 / 2$, and $S=0$. As discussed previously, Fig. 2 shows that the ground states of a nanotube quantum dot in regions I and III are spin degenerate, whereas the electronic state in region II can be spin singlet or multiplet depending on the exact electron numbers. The experimental observation is therefore consistent with the spin-filling schemes outlined above, and indicates that both spin-filling scenarios are realized in a nanotube quantum dot. Moreover, the excited states responsible for the inelastic cotunneling features in regions II and IV can be accounted for by the triplet states in Fig. 2(c), in a manner consistent with experimentally observed magnetic-field dependence.

The analysis of the experimental data further allows the quantitative determination of $\Delta, \delta, U, J$, and $\delta U$. This 
task is accomplished by equating the theoretically determined energies [29] with their experimentally measured values and solving the resulting set of equations. In both theory and experiment, the sizes of the conductance-gap regions I and III are equal, yielding equations for three independent addition energies $e V_{\mathrm{I}-\mathrm{IV}}$ and the energies of the inelastic cotunneling features $e V_{\text {in }}$ [29]. In addition, $\Delta$ can be fixed using the experimentally determined nanotube length $(L)$ since $\Delta=h \nu_{\mathrm{F}} / 2 L$ where $h$ is Plank's constant and $\nu_{\mathrm{F}}$ is the Fermi velocity $[6,13]$.

Values of the energy parameters obtained from our analysis are presented in Fig. 3. The dotted lines in Figs. 2(a) and 2(b) indicate the positions of transport features calculated using these parameters, and they confirm that the shell-filling model explains the experimental data extremely well. As shown in Fig. 3, the parameter values determined from five distinct four-electron periods of the same nanotube device remain approximately constant although they do fluctuate as the electron number on the quantum dot is varied. These small fluctuations, which may be caused by disorder, account for the experimentally observed variation in ground state $S$ value in region II with the electron number. The averaged parameter values, normalized to $\Delta=h \nu_{\mathrm{F}} / 2 L=8.4 \mathrm{meV}$, are given by $U / \Delta=0.26, \quad J / \Delta=0.12, \quad \delta U / \Delta=0.04$, $\delta / \Delta=0.27$. Similar values for $J / \Delta \sim 0.1-0.2$ and $\delta U / \Delta \sim 0.05-0.1$ were found from other nanotube devices with $G_{\mathrm{av}} \sim(1-2) e^{2} / h$ as well, as shown in Fig. 3. These values for $J$ and $\delta U$ compare favorably with the theoretically predicted values for $(10,10)$ armchair nanotubes of $J / \Delta=0.22$ and $\delta U / \Delta=0.11$ [22].

It is noteworthy that the value of $U / \Delta \sim 0.26$ determined from our analysis is an order of magnitude smaller than what is typically observed in nanotube dots with $G_{\text {av }} \ll e^{2} / h$. This small value of $U$ is consistent with previous transport studies on semiconductor quantum dots with high conductance [30], and it plays a critical role for the clear observation of the transport features in Fig. 2 and hence the determination of the relatively small energy scales such as $\delta U, J$, and $\delta$. The nonzero value of $\delta$ determined from our analysis suggests, on the other hand, that the subband degeneracy is broken in a nanotube quantum dot. The exact value of $\delta$ is expected to vary randomly in different nanotube devices depending on the boundary conditions at the nanotube-metal interface [22]. Extensive analyses of the shell-filling model with $J / \Delta, \delta U / \Delta$, and $U / \Delta$ shown in Fig. 3 indicate, however, that the fourelectron periodicity should be preserved in approximately $70 \%$ of these nanotube devices, providing an explanation for the robust four-electron periodicity for nanotube devices with $G_{\mathrm{av}} \sim(1-2) e^{2} / h$.
We acknowledge C.M. Lieber, M. Tinkham, B.I. Halperin, and J.H. Hafner for discussions. This work is supported by NSF, DARPA, the Dreyfus Foundation, the Packard Foundation, the Research Corporation, and Harvard University. M. B. is partially supported by the Department of Physics, Harvard University. W.L. and M.B. contributed equally to this work.

*To whom correspondence should be sent. Email address: HPark@ chemistry.harvard.edu

[1] H. Park et al., Nature (London) 407, 57 (2000).

[2] D. C. Ralph et al., Phys. Rev. Lett. 78, 4087 (1997).

[3] D. L. Klein et al., Nature (London) 389, 699 (1997).

[4] H. Park et al., Appl. Phys. Lett. 75, 301 (1999).

[5] S. J. Tans et al., Nature (London) 386, 474 (1997).

[6] M. Bockrath et al., Science 275, 1922 (1997).

[7] S. J. Tans et al., Nature (London) 394, 761 (1998).

[8] D. H. Cobden et al., Phys. Rev. Lett. 81, 681 (1998).

[9] A. Bezryadin et al., Phys. Rev. Lett. 80, 4036 (1998).

[10] M. Bockrath et al., Nature (London) 397, 598 (1999).

[11] J. Nygard et al., Nature (London) 408, 342 (2000).

[12] M. Bockrath et al., Science 291, 283 (2001).

[13] W. Liang et al., Nature (London) 411, 665 (2001).

[14] H. Grabert and M. H. Devoret, Single Charge Tunneling (Plenum, New York, 1992).

[15] L. I. Glazman and M. E. Raikh, JETP Lett. 47, 452 (1988).

[16] Y. Meir et al., Phys. Rev. Lett. 70, 2601 (1993).

[17] D. Goldhaber-Gordon et al., Nature (London) 391, 156 (1998).

[18] S. M. Cronenwett et al., Science 281, 540 (1998).

[19] S. Sasaki et al., Nature (London) 405, 764 (2000).

[20] W. G. van der Wiel et al., Science 289, 2105 (2000).

[21] S. De Franceschi et al., Phys. Rev. Lett. 86, 878 (2001).

[22] Y. Oreg et al., Phys. Rev. Lett. 85, 365 (2000).

[23] S. Tarucha et al., Phys. Rev. Lett. 77, 3613 (1996).

[24] L. P. Kouwenhoven et al., Science 278, 1788 (1997).

[25] S. Tarucha et al., Phys. Rev. Lett. 84, 2485 (2000).

[26] J. H. Hafner et al., Chem. Phys. Lett. 296, 195 (1998).

[27] M. Pustilnik et al., Phys. Rev. Lett. 84, 1756 (2000).

[28] C. T. White and T. N. Todorov, Nature (London) 393, 240 (1998).

[29] The electron addition energies $\left(e V_{\mathrm{I}-\mathrm{IV}}\right)$ and the energy of the cotunneling feature are given in terms of $\Delta, \delta, U$, $J$, and $\delta U$ by $e V_{\mathrm{I}}=e V_{\mathrm{III}}=U+J+\delta U(U+\delta)$, $e V_{\mathrm{II}}=U+\delta-\delta U(U+2 J-\delta+\delta U), \quad e V_{\mathrm{IV}}=$ $U+\Delta-\delta U-\delta(U+\Delta-\delta U-\delta), \quad$ and $e V_{\text {in }}$ $($ Region IV $)=\Delta-J-\delta U-\delta(\Delta-J-\delta U-\delta)$. Here the formulas pertain to the situation where the region II has $S=0(S=1)$.

[30] L.W. Molenkamp et al., Phys. Rev. Lett. 75, 4282 (1995). 